\title{
P03-13-4 Poster session
}

\section{Characterization of response to acute and chronic arsenic toxicity in CD34high- enriched epidermal stem cells}

\author{
Yuanyuan Xu, Yuxin Hu, Xiafang Wu, Ru Sun, Xin Fang, Huihui Wang, Jingbo Pi \\ School of Public Health, China Medical University, China
}

Arsenic has been successfully used as an anticancer agent against acute promyelocytic leukemia and some solid tumors, whereas it is identified as a human carcinogen. The skin is an important target organ of chronic arsenic toxicity, which limits medical use of arsenic agent. Previous studies suggest that stem cells play a key role in arsenic poisoning. In the present study, we isolated CD34high-enriched cells from HaCaT human keratinocytes by magnetic-activated cell sorting, and analyzed stem/progenitor cell characteristics, resistance to acute arsenic cytotoxicity, and sensitivity to arsenicinduced malignant transformation in these cells. We found that expression of stem cell markers, CD34, p63, K5, K14, OCT4 and SHH, was significantly higher in CD34high cells than in CD34low cells. CD34high cells exhibited a higher ability to form holoclones, which could undergo serial passage for more than 3 generations. Median lethal dose (LD50), anti-apoptotic capacity, and arsenic efflux ability in response to acute arsenic exposure (20 to 80 micromole arsenite, 24h) were all significantly higher in CD34high cells than in CD34low cells $(\mathrm{p}<0.05)$. NRF2 with its downstream genes (ABCC1, ABCG2, HMOX1, and GCLC) was highly expressed in CD34high cells compared with CD34low cells ( $\mathrm{p}<$ 0.05). Stable knockdown of NRF2 abolished hyper-resistance to acute arsenic toxicity and holocolone forming ability of CD34high cells. When continuously exposed to a low level of sodium arsenite (100 nanomole) for 12 weeks, CD34high cells showed epithelial-mesenchymal transitions (EMT). However, there was no significant change in control mature cells. After 30 weeks of arsenic exposure, HaCaT cells exhibited malignant phenotypes and a significant elevation in CD34high subpopulation $(\mathrm{p}<0.05)$. Stable knockdown of NRF2 abolished the above phenotypes induced by arsenic $(\mathrm{p}<0.05)$. In summary, CD34high cells exhibit a stem/progenitor-like phenotype. These skin epithelial stem/progenitor-like cells are more resistant to acute arsenic toxicity and more sensitive to arsenic-induced malignant transformation than mature cells, which is closely related to their innate higher expression of NRF2. These data provide a new information on the mechanism of skin damage by arsenic. 